Check for updates

Cite this: RSC Adv., 2019, 9, 3020

\title{
The neutralization of heparan sulfate by heparin- binding copolymer as a potential therapeutic target
}

\author{
Bartlomiej Kalaska, (D) Joanna Miklosz, (D) ${ }^{a}$ Kamil Kamiński, ${ }^{\mathrm{b}}$ Bogdan Musielak, ${ }^{\mathrm{b}}$ \\ Shin-Ichi Yusa, ${ }^{c}$ Dariusz Pawlak, ${ }^{a}$ Maria Nowakowska, ${ }^{b}$ Krzysztof Szczubiałka*b \\ and Andrzej Mogielnicki (D) *a
}

Besides regulating ligand-receptor and cell-cell interactions, heparan sulfate (HS) may participate in the development of many diseases, such as cancer, bacterial or viral infections, and their complications, like bleeding or inflammation. In these cases, the neutralization of HS could be a potential therapeutic target. The heparin-binding copolymer (HBC, PEG41-PMAPTAC53) was previously reported by us as a fully synthetic compound for efficient and safe neutralization of heparins and synthetic anticoagulants. In a search for molecular antagonists of HS, we examined the activity of HBC as an HS inhibitor both in vitro and in vivo and characterized $\mathrm{HBC} / \mathrm{HS}$ complexes. Using a colorimetric Azure A method, isothermal titration calorimetry and dynamic light scattering techniques we found that HBC binds HS by forming complexes below $200 \mathrm{~nm}$ with less than $1: 1$ stoichiometry. We confirmed the HBC inhibitory effect in rats by measuring activated partial thromboplastin time, prothrombin time, anti-factor Xa activity, antifactor Ila activity, and platelet aggregation. HBC reversed the enhancement of all tested parameters caused by HS demonstrating that cationic synthetic block copolymers may have a therapeutic value in various disorders involving overproduction of HS.

Received 26th November 2018

Accepted 15th January 2019

DOI: $10.1039 / \mathrm{c} 8 \mathrm{ra0} 0724 \mathrm{k}$

rsc.li/rsc-advances close to heparin, shows anticoagulative properties, although weaker than those of the latter. ${ }^{1}$ Like in the case of heparins, the degree of sulfation of HS may vary significantly and both low- $^{7}$ and highly-sulfated ${ }^{8}$ forms of HS have been found depending on the tissue of origin or in some pathologies. ${ }^{9-11}$

Unfortunately, HS also participates in many pathological processes. HS may play a crucial role in cancer progression, angiogenesis, and metastasis ${ }^{12,13}$ showing both tumor progressing ${ }^{14}$ or tumor inhibiting activity. ${ }^{15} \mathrm{HS}$ was present in the fluid of an ascitic form of a chemically-induced pancreatic ductal adenocarcinoma in the Syrian golden hamster. Moreover, plasma containing the fluid showed markedly prolonged coagulation times, indicating that HS may be largely responsible for the bloody ascites. ${ }^{16}$ More recently it was found that high serum HS concentration was responsible for the poor treatment outcome with epidermal growth factor receptortyrosine kinase inhibitors in patients with non-small cell lung cancer. ${ }^{17}$ Since in this study HS concentrations were significantly higher in patients with progressive cancer than in those with a non-progressive form of cancer, HS was proposed as a candidate biomarker for treatment resistance.

Pathologically increased plasma levels of endogenous HS may also be responsible for serious bleeding. Palmer and coauthors have identified HS in the blood of a patient with multiple myeloma who had lethal bleeding. ${ }^{18}$ The authors concluded that recognition of the existence of endogenous anticoagulant inhibitors in bleeding patients could be crucial
${ }^{a}$ Department of Pharmacodynamics, Medical University of Bialystok, Mickiewicza 2c, 15-089 Bialystok, Poland. E-mail: amogiel@umb.edu.pl

${ }^{b}$ Faculty of Chemistry, Jagiellonian University, Gronostajowa 2, 30-387 Krakow, Poland. E-mail: szczubia@chemia.uj.edu.pl

'Department of Applied Chemistry, Graduate School of Engineering, University of Hyogo, Himeji, Hyogo, Japan 
because of the potential for treatment with proper antidotes. Recently, it has been shown that patients with untreated mucopolysaccharidosis (MPS) had higher levels of HS in blood in comparison to age-matched controls. ${ }^{19}$ Tolar et al. found that increased levels of GAGs including HS were responsible for the increased risk of life-threatening pulmonary bleeding. ${ }^{20}$

HSPGs have been found to be the components of the attachment receptors of many viruses. ${ }^{21}$ It has been shown that the interaction of dengue virus (DV) with human cells is mediated by HSPGs, ${ }^{22}$ and a highly sulfated type of HS is a cellular receptor utilized by DV envelope protein to bind to target cells. ${ }^{23}$ Other ligands of this receptor such as heparin or the polysulfonated pharmaceutical suramin prevented DV infection, indicating that the interaction with HS is a critical determinant of infectivity. Furthermore, acute HS levels were significantly higher in dengue patients and decreased at convalescence. ${ }^{24}$ The authors concluded that developing pharmaceuticals that inhibit target cell binding may be an effective strategy for treating flavivirus infections. ${ }^{23}$ HS-containing receptors are also involved in the entry of many other viruses causing both severe diseases (human immunodeficiency virus (HIV), ${ }^{25}$ hepatitis C virus (HCV), ${ }^{26}$ herpes simplex virus (HSV), ${ }^{27}$ Japanese encephalitis virus), ${ }^{28}$ mild pathologies (Sindbis virus ${ }^{29}$ foot-and-mouth disease virus, ${ }^{30}$ coxsackievirus A), ${ }^{31}$ and nonpathogenic ones (human foamy virus). ${ }^{32}$ Recently, it was also shown that blocking the interaction of HS with Ebola virus leads to significant inhibition of basolateral infection of epithelial cells. $^{33}$

Myocardial dysfunction in sepsis has been linked to inflammation caused by pathogen-associated and host dangerassociated molecular patterns, which include soluble HS. ${ }^{34}$ Indeed, the administration of endotoxin from a Gram-negative bacterium (Escherichia coli) to pigs resulted in increased serum levels of HS, signalizing a shedding of the glycocalyx. ${ }^{35}$ The experiments in HL-1 cardiomyocytes showed that compounds antagonizing HS might have the potential for further development as broad-spectrum anti-inflammatory agents in sepsisinduced myocardial inflammation and dysfunction. ${ }^{34}$

All of these findings indicate that HS could be a novel therapeutic target in the treatment of cancer, viral infections, bleeding, inflammatory processes, MPS and possibly other diseases. Therefore, both low-molecular-weight and macromolecular compounds have been developed as the inhibitors of various activities of HS or its biosynthesis. For example, surfen, a small molecule capable of binding to HS, was found to inhibit HS-dependent binding, and signaling by fibroblast growth factor 2 (FGF2), infection by HSV-1, cell attachment, and endothelial sprouting stimulated by vascular endothelial growth factor and FGF2. ${ }^{36}$ Its interaction with HS also resulted in strong and general inhibition of differentiation and promotion of pluripotency in mouse embryonic stem cells. ${ }^{37}$ In a very recent study surfen and its derivative, oxalyl surfen, showed potential in the inhibition of tau hyperphosphorylation, which was found to strongly correlate with the progression and memory loss in Alzheimer's disease. ${ }^{38}$ It was suggested that preventing tau hyperphosphorylation was due to surfeninduced alterations in HS synthesis, metabolism, structure, and stability. Moreover, compounds that inhibit HS-protein interactions have considerable potential as anti-inflammatory therapeutics. $^{39}$ Also, glucosamine analogs which inhibit HS biosynthesis have been proposed as possible drugs in the treatment of amyloid diseases. ${ }^{40}$ However, inhibiting HS synthesis may result in unpredictable adverse effects taking into account the multiple physiological roles of HS.

In this study, we have focused on the possibility of inhibition of the anticoagulant activity of HS, particularly because there are no reports on the reversal of HS-induced bleeding. This action of HS was previously neutralized by protamine. ${ }^{\mathbf{4 1}}$ However, the inhibition was weaker than that of unfractionated heparin (UFH), especially when measured by anti-factor Xa (anti-fXa) activity. Moreover, taking into account the safety issues related to protamine use, it is not a good therapeutic candidate. ${ }^{42}$ We have recently developed a heparin-binding copolymer (HBC, PEG41-PMAPTAC53) well-tolerated by rats and mice, which may constitute an alternative to protamine in the efficient neutralization of UFH. Importantly, we found that HBC also reverses anticoagulation caused by low-molecularweight heparins (LMWHs), ${ }^{43}$ fondaparinux, ${ }^{43}$ and sulfonated synthetic polymers. ${ }^{44}$ Here, our aim was to check whether HBC may bind and neutralize HS in the living organism. We used the sample of HS as a model to show potential of HBC as a new therapeutic for various disorders involving increased concentration of HS.

\section{Materials and methods}

\section{Materials}

HS (95.95\%) from 6C USA Inc was used as received. 4-Cyano-4(phenylcarbonothioylthio)pentanoic acid was synthesized according to the method reported by Mitsukami et al. ${ }^{\mathbf{4 5}}$ Poly(ethylene glycol)methyl ether (average molecular weight $2000 \mathrm{Da}$ ) from Aldrich was used as received without further purification. Poly(ethylene glycol)4-cyano-4(phenylcarbonothioylthio)pentanoate (PEG41-CTA) was synthesized according to the literature with slight modification. ${ }^{46}$ 3-(Methacryloylamino)propyl trimethylammonium chloride (MAPTAC, $50 \mathrm{wt} \%$ in water) from Aldrich was passed through an inhibitor removal column. 4,40-Azobis(4cyanopentanoic acid; V-501, 98\%) from Wako Pure Chemical was used as received without further purification. Azure A chloride (Fluka standard) was purchased from Fluka (Switzerland). Water was purified with a Millipore Milli-Q system. Other reagents were used as received.

\section{HBC preparation and characterization}

The preparation, recovery, and characterization of HBC have been described previously. ${ }^{43}$ Briefly, MAPTAC (5.53 g, 25.0 mmol), V-501 (69.2 mg, $0.247 \mathrm{mmol}$ ), and PEG41-CTA (1.13 g, $0.501 \mathrm{mmol})$ were dissolved in water $(41.0 \mathrm{~mL})$. The mixture was degassed by purging with Ar gas for $30 \mathrm{~min}$. Reversible Addition-Fragmentation chain Transfer (RAFT) polymerization was carried out at $70^{\circ} \mathrm{C}$ for $5 \mathrm{~h}$. The polymerization mixture was 
dialyzed against pure water for two days. HBC was recovered using a freeze-drying technique $(5.58 \mathrm{~g}, 83.8 \%)$.

\section{In vitro anticoagulant properties of HS}

The anticoagulant properties of HS were analyzed by measuring activated partial thromboplastin time (aPTT), prothrombin time (PT), and anti-fXa and anti-factor IIa (anti-fIIa) activity. In brief, $200 \mu \mathrm{L}$ of pooled rat plasma were mixed with $20 \mu \mathrm{L}$ of HS (0.005, 0.01, 0.05, $0.1 \mathrm{mg} \mathrm{mL}^{-1}$ of pooled plasma). After $5 \mathrm{~min}$ of incubation $\left(37^{\circ} \mathrm{C}\right)$, coagulation parameters were measured according to the manufacturer instructions. aPTT and PT were analyzed with an optical method (Coag Chrom 4000, Bio-Ksel) and routine laboratory reagents (Bio-Ksel). Anti-fXa and antifIIa activities were determined by chromogenic assays (Dynex Tech.) and the manufacturer instructions (Sekisui Diagnostics).

\section{In vitro binding of $\mathrm{HS}$ by $\mathrm{HBC}$}

The ability of HBC to bind HS was assessed with a colorimetric method using Azure A, as described previously ${ }^{43,44,47}$ Briefly, HS forms a complex with Azure A. Dimers of Azure A absorb at $513 \mathrm{~nm}$ while the monomeric forms of Azure A absorb at $630 \mathrm{~nm}$. The addition of HS results in the disruption of Azure A dimers and consequently increases $630 \mathrm{~nm}$ and decreases $513 \mathrm{~nm}$ absorption. The initial concentration of HS in the mixture was $0.2 \mathrm{~g} \mathrm{~L}^{-1}$ whereas the concentration of the polycation added was $1.6 \mathrm{~g} \mathrm{~L}^{-1}$. The volumes were mixed in such a way that phosphate buffer solution (PBS) and a polycation solution was added to $0.5 \mathrm{~mL}$ of $\mathrm{HS}$ so that the total volume of the sample was $1 \mathrm{~mL}$.

\section{In vitro neutralization of $\mathrm{HS}$ by $\mathrm{HBC}$}

The ability of HBC to neutralize HS was assessed by measuring aPTT, PT, and anti-fXa and anti-fIIa activity, as described previously. ${ }^{43,44,47}$ Briefly, $10 \mu \mathrm{L}$ of $\mathrm{HS}\left(0.05 \mathrm{mg} \mathrm{mL}^{-1}\right.$ of plasma) was mixed with $200 \mu \mathrm{L}$ of pooled plasma. After $2 \mathrm{~min}$ and $30 \mathrm{~s}$ of incubation, $10 \mu \mathrm{L}$ of a solution containing increasing concentrations of HBC $\left(0.0125,0.025,0.0375,0.05 \mathrm{mg} \mathrm{mL}^{-1}\right.$ of pooled plasma; mass ratios $0.25: 1,0.5: 1,0.75: 1,1: 1$ ) was added. After $2 \mathrm{~min}$ and $30 \mathrm{~s}$ of incubation, the aPTT, PT, and anti-fXa and anti-fIIa activity were automatically determined according to the methods described above.

\section{Dynamic light scattering (DLS) and zeta potential measurements}

The size of complexes formed by HBC and HS was studied with the DLS technique in serum and PBS. HS was dissolved in PBS or bovine serum $\left(0.4 \mathrm{mg} \mathrm{mL} \mathrm{m}^{-1}\right)$. To that mixture, the $\mathrm{HBC}$ solutions in PBS buffer were added $\left(12 \mathrm{mg} \mathrm{mL}^{-1}\right)$, and the formation of complexes was monitored by DLS and zeta potential using a Zetasizer Nano-ZS (Malvern Instruments, UK).

\section{Isothermal titration calorimetry (ITC) measurements}

ITC measurements were made using Malvern MICROCAL PEAQ-ITC. HBC was dissolved in PBS; its concentration was $8 \mathrm{~g}$ $\mathrm{L}^{-1}\left(0.001078 \mathrm{~mol} \mathrm{~L}^{-1}\right)$. HS was also dissolved in PBS in the concentration of $0.5 \mathrm{~g} \mathrm{~L}^{-1}$. The measurement temperature was $25{ }^{\circ} \mathrm{C}$ and the mixing speed of $750 \mathrm{rpm}$. The measuring chamber contained $270 \mu \mathrm{L}$ of HS solution to which 19 portions of HBS solution, each $2 \mu \mathrm{L}$, were added.

\section{Animals}

Male Wistar rats obtained from the Centre of Experimental Medicine in the Medical University of Bialystok were bred in a $12 \mathrm{~h} \mathrm{light/dark}$ cycle in a humidity- and temperaturecontrolled room in specific pathogen-free conditions. They allowed having ad libitum access to standard chow and sterilized tap water. All the procedures involving animals were approved by Local Ethical Committee on Animal Testing (Permit Number 60/2018) and conducted according to Directive 2010/63/EU of the European Parliament and the Council on the protection of animals, ARRIVE guidelines, and the national laws.

\section{In vivo neutralization of $\mathrm{HS}$ by $\mathrm{HBC}$}

Thirty-three male Wistar rats weighing 182-212 g were randomly assigned to 3 groups, anesthetized by an intraperitoneal injection of pentobarbital ( $\left.45 \mathrm{mg} \mathrm{kg}^{-1}\right)$, and placed in a supine position on a heated operating table. Rats were intravenously injected into the right femoral vein with HBC $(4.5 \mathrm{mg}$ $\left.\mathrm{kg}^{-1}\right) 5 \mathrm{~min}$ after injection with HS $\left(6 \mathrm{mg} \mathrm{kg}^{-1}\right)$. Twenty minutes after HS administration, the blood samples were collected from the heart and drawn into $3.13 \%$ trisodium citrate. Neutralization of HS by HBC was monitored by measuring aPTT, PT, antifXa and anti-fIIa activity, and platelet aggregation. Coagulation parameters were determined as described above whereas platelet aggregation was measured after incubation of sodium citrate-anticoagulated blood with $0.9 \% \mathrm{NaCl}$ solution (volume ratio $1: 1$ ) for $20 \mathrm{~min}$ at $25^{\circ} \mathrm{C}$ and then for $15 \mathrm{~min}$ at $37^{\circ} \mathrm{C}$. The changes in impedance registered as aggregation curve were recorded for $6 \mathrm{~min}$ after collagen addition $\left(7.5 \mu \mathrm{g} \mathrm{mL}^{-1}\right)$ using a Chrono-log aggregometer (Chrono-log Corp.). Four parameters were automatically calculated: maximal extension (MaxA), lag phase (Lag), the slope of platelet aggregation (Slp), and area under the curve (AUC). The white blood cell count (WBC) was automatically performed using an animal blood counter (scil Vet abc Plus, Horiba, France).

\section{Statistical analysis}

The data is shown as median with lower and upper limits or mean \pm SD and analyzed using the Mann-Whitney test or unpaired Student's $t$-test, depending on the normal distribution results. Results were analyzed and graphically presented using GraphPad Prism 6 software (USA). $P$ values less than 0.05 were considered significant.

\section{Results}

\section{The anticoagulant properties of HS in rat plasma}

HS concentration-dependently and maximally increased aPTT and showed both anti-fXa and anti-fIIa activities. The maximum anticoagulant effect occurred at $0.05 \mathrm{mg} \mathrm{mL}^{-1}$ as measured by 
A

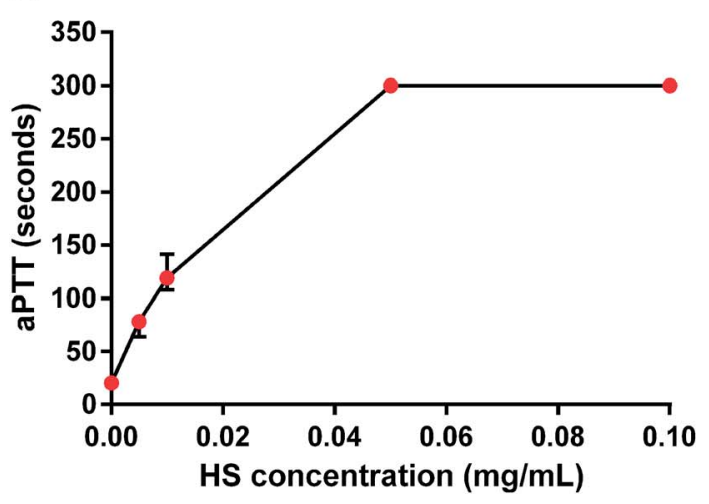

C

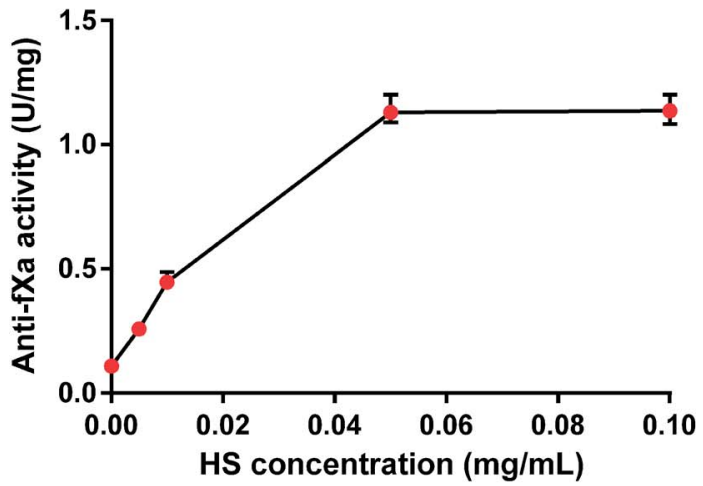

B

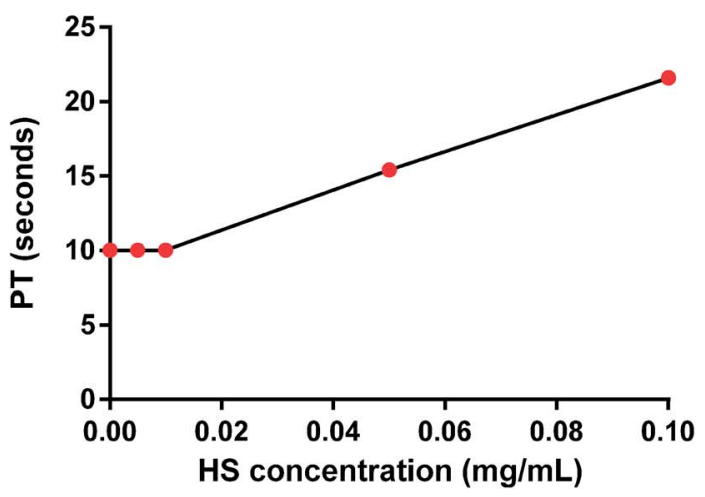

D

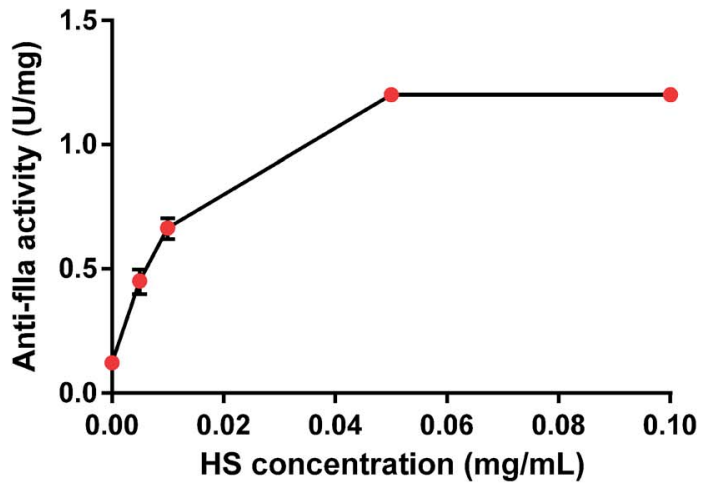

Fig. 1 Anticoagulant properties of heparan sulfate (HS) in rat plasma. Effects of HS were monitored by measuring activated partial thromboplastin time (aPTT, A), prothrombin time (PT, B), anti-factor Xa (anti-fXa, C) and anti-factor Ila (anti-flla) activity (D). Results are shown as median values with lower and upper limits.

aPTT, anti-fXa and anti-fIIa activity tests, while PT was prolonged two-fold for $\mathrm{HS}$ at $0.1 \mathrm{mg} \mathrm{mL} \mathrm{m}^{-1}$ (Fig. 1). We chose $0.05 \mathrm{mg} \mathrm{mL}^{-1}$ concentration of $\mathrm{HS}$ for further in vitro studies.

The binding and the neutralization of HS by HBC in phosphate-buffered saline and rat plasma

From among a large panel of polymers we have previously studied as the inhibitors of $\mathrm{UFH}^{43,47-51}$ and synthetic polymeric anticoagulants $^{44}$ we have selected $\mathrm{HBC}$ as a potential HS inhibitor (Fig. 2).

HBC is a block copolymer composed of a cationic block of poly(3-(methacryloylamino)propyl trimethylammonium chloride) (PMAPTAC) and a neutral block of poly(ethylene glycol) (PEG). HBC was synthesized using RAFT allowing for the preparation of the block polymers with very low molecular weight dispersity. The molecular weight of HBC and its dispersity index<smiles>CC(C)(CCC(=O)OCCOC(C)(C)C)CC(C)(C)C(=O)NCCC[N+](C)(C)C</smiles>

Fig. 2 The chemical structure of heparin-binding copolymer (HBC). 


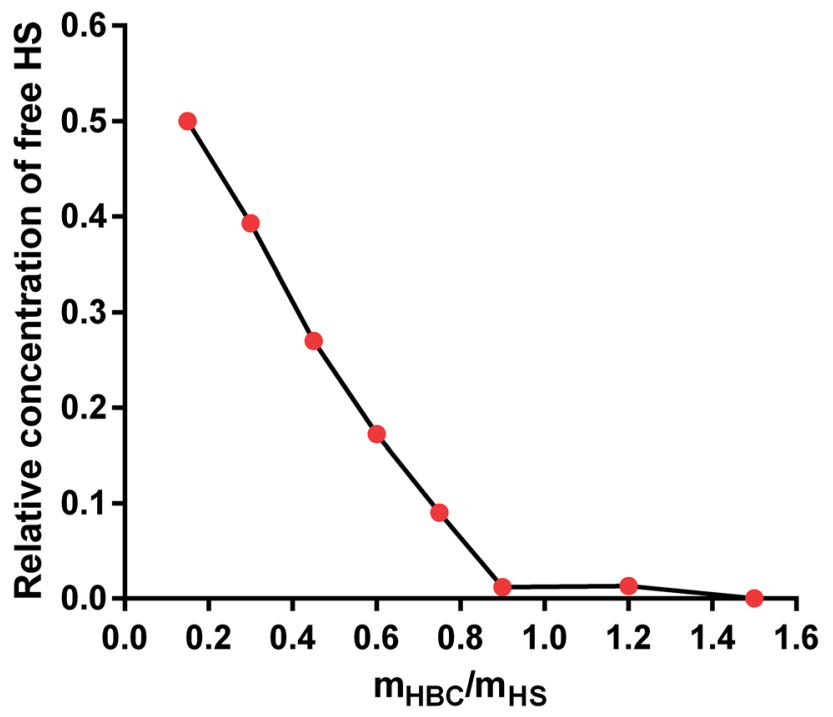

Fig. 3 The binding of heparan sulfate (HS) by heparin-binding copolymer (HBC) in phosphate buffer solution (PBS).

were found to be $14 \mathrm{kDa}$ (nuclear magnetic resonance, NMR) and 1.02 (gel permeation chromatography, GPC), respectively. The molecular weight of HBC was calculated based on the integral intensity ratios of signals of the methylene protons in the PEG block and the pendant methylene protons in the PMAPTAC block and assuming the molecular weight of the PEG block of 2000 Da provided by the manufacturer. HBC showed superior in vitro and in vivo properties as a UFH inhibitor compared to other studied polymers and protamine..$^{43,47-51}$ To find out if HS is negatively charged enough to form a complex with cationic HBC, we used a colorimetric method using Azure A, a cationic dye. The experiment revealed that HBC completely bound HS in PBS with an optimal ratio of $0.9 \mathrm{mg}$ of the copolymer for $1 \mathrm{mg}$ of HS (Fig. 3).

For further analysis of the effectiveness of $\mathrm{HBC}$, we studied the in vitro neutralization of HS measured as aPTT, PT, anti-fXa activity, and anti-fIIa activity in rat plasma. The results of the coagulation tests indicated that the full neutralization of HS required an $\mathrm{HBC}$ to $\mathrm{HS}$ ratio of about $0.75: 1$. Anti-fIIa activity in the presence of HS was decreased by HBC even below the desired normal value (Fig. 4). We chose 0.75 : 1 (HBC : HS) mass ratio for further in vivo studies.

\section{The neutralization of $\mathrm{HS}$ by $\mathrm{HBC}$ in rats}

We used the same coagulation parameters to determine the in vivo inhibitory potency of HBC on HS in Wistar rats as in the in vitro studies. HBC completely neutralized HS as assessed by aPTT, PT, and anti-IIa assays (Fig. 5). Anti-fXa assay showed

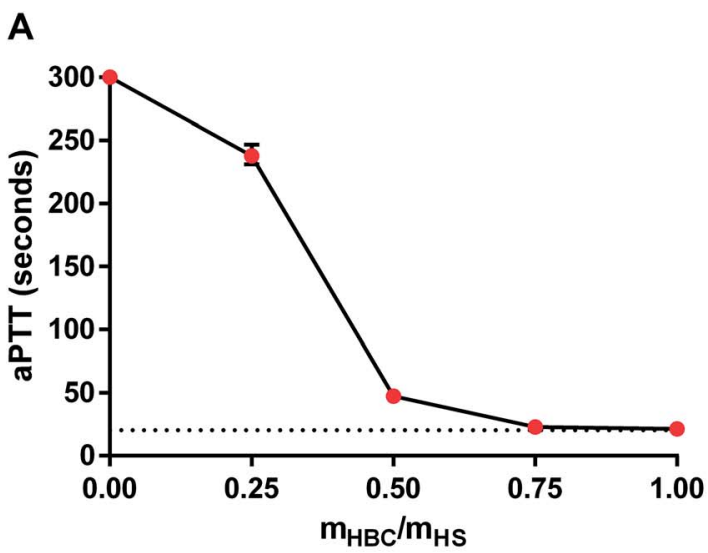

B

C
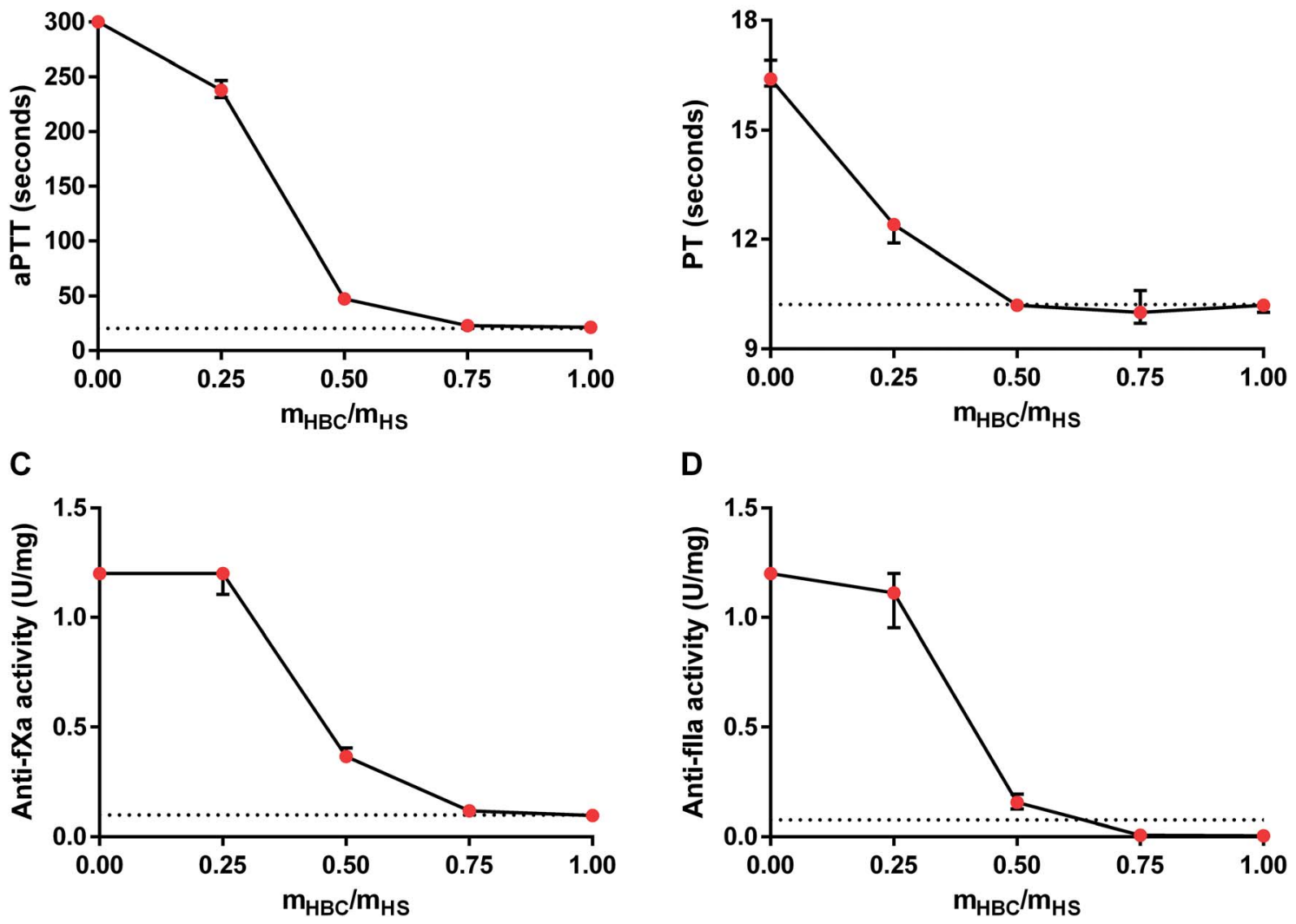

D

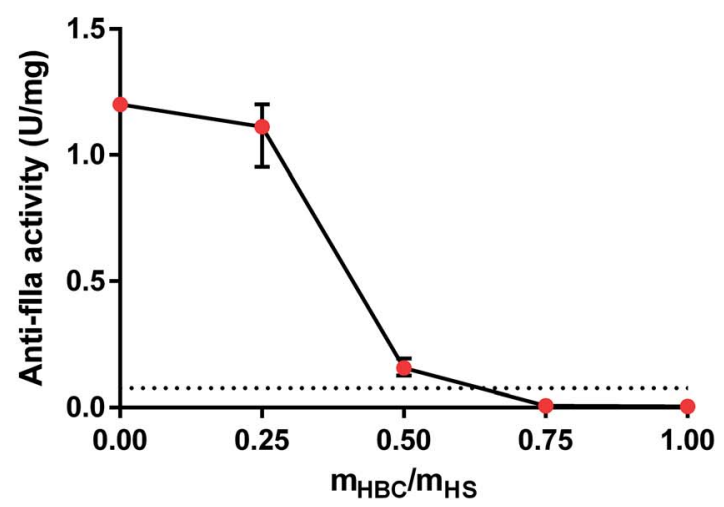

Fig. 4 Neutralization of heparan sulfate (HS) by heparin-binding copolymer (HBC) in rat plasma. Neutralization was monitored in vitro by measuring activated partial thromboplastin time (aPTT, A), prothrombin time (PT, B), anti-factor Xa (anti-fXa) activity (C), and anti-factor Ila (anti$\mathrm{fXa}$ ) activity (D). Results are shown as median values with lower and upper limits. Dotted lines indicate normal values for coagulation parameters of rat plasma. 
A

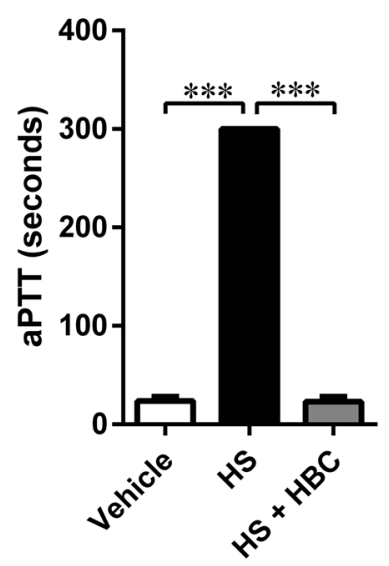

C

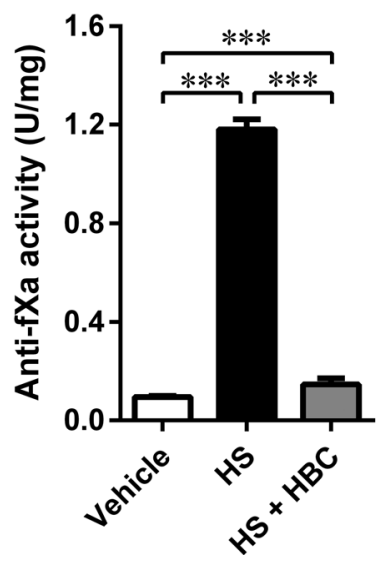

B

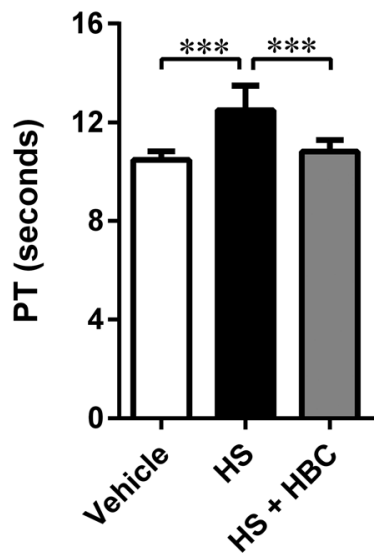

D

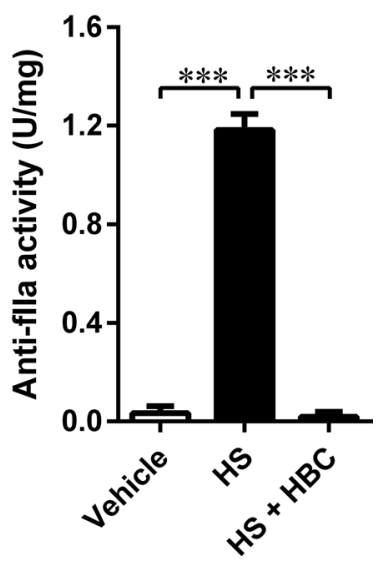

Fig. 5 Neutralization of heparan sulfate (HS) $\left(6 \mathrm{mg} \mathrm{kg}^{-1}, 1 \mathrm{~mL} \mathrm{~kg}^{-1}\right)$ by heparin-binding copolymer (HBC) $\left(4.5 \mathrm{mg} \mathrm{kg}^{-1}, 1 \mathrm{~mL} \mathrm{~kg}^{-1}\right.$ by weight) in Wistar rats. Neutralization was assessed by measuring activated partial thromboplastin time (aPTT, A), prothrombin time (PT, B), antifactor Xa activity (anti-fXa, C), and anti-factor lla activity (anti-fXa, D). $* * * p<0.001$, unpaired Student's $t$-test. Results are shown as mean \pm $\mathrm{SD}$.

more than $90 \%$ neutralization of HS activity by HBC. HBC had no procoagulant properties as observed in the in vitro anti-IIa assay.

In the same animal model, we investigated the effect of HS alone and followed by HBC on platelet aggregation. HS induced statistically significant activation of collagen-induced platelet aggregation expressed as MaxA and AUC. HBC administered after HS did not affect the platelet aggregation compared to PBSinjected rats, but significantly reduced the slope of platelet aggregation curve compared to HS-injected rats (Fig. 6). HBC did not potentiate the increase of WBC induced by HS $\left(2.6 \pm 0.7 \times 10^{3}\right)$ $\mathrm{mm}^{-3}, 3.7 \pm 0.7 \times 10^{3} / \mathrm{mm}^{-3}$, and $4.1 \pm 0.7 \times 10^{3} / \mathrm{mm}^{-3}$ in the vehicle, HS, and HS + HBC, respectively; $p<0.05$ vehicle $v s$. HS).

The size of HS-HBC complexes and heat of HS binding by HBC

Using DLS technique, we found that the diameter of the objects formed in the PBS solution of HS and in the mixtures of PBS

A

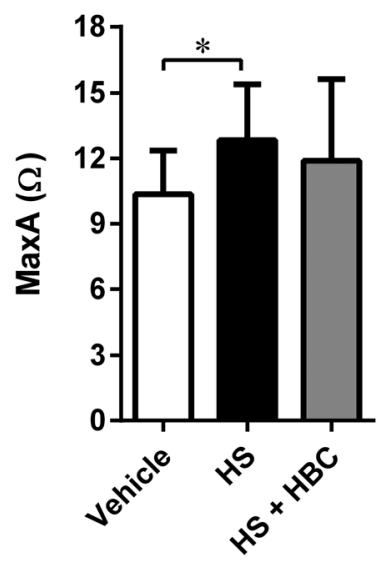

B

C

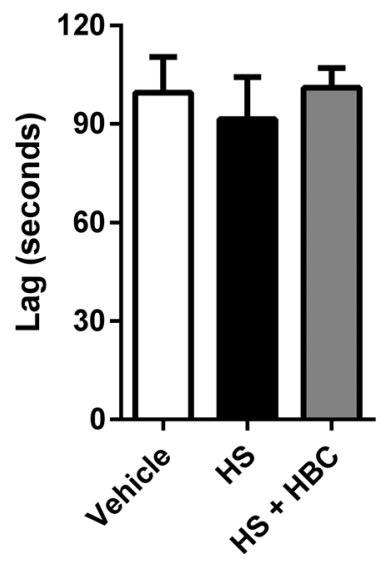

D

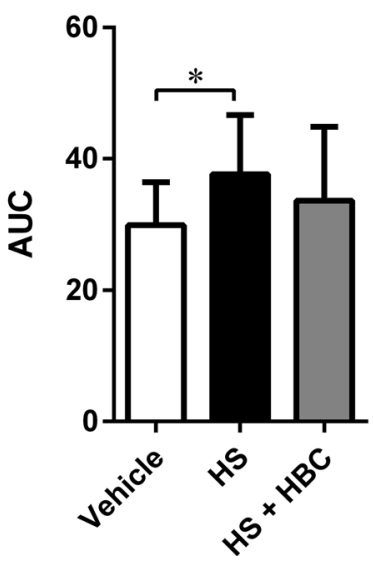

Fig. 6 Neutralization of heparan sulfate (HS) $\left(6 \mathrm{mg} \mathrm{kg}^{-1}, 1 \mathrm{~mL} \mathrm{~kg}^{-1}\right)$ by heparin-binding copolymer (HBC) $\left(4.5 \mathrm{mg} \mathrm{kg}^{-1}, 1 \mathrm{~mL} \mathrm{~kg}^{-1}\right)$ in Wistar rats. Neutralization was assessed by measuring platelet aggregation expressed as the maximal extension (MaxA, A), the slope of platelet aggregation (Slp, B), lag time (Lag, C), and area under the curve (AUC, D) in rats. ${ }^{*} p<0.05$, unpaired Student's $t$-test. Results are shown as mean \pm SD.

solutions of $\mathrm{HS}$ and $\mathrm{HBC}$ at $\mathrm{HBC} / \mathrm{HS}$ mass ratios from 0.3 to 1.5 was below $500 \mathrm{~nm}$ (Fig. 7, Table 1).

Both HS and HBC are strong polyelectrolytes therefore one may expect that they form complexes with various proteins present in serum. ${ }^{52}$ Indeed, we observed that HBC formed complexes of very different sizes with serum proteins as indicated by the three maxima present in the DLS spectrum of HBC in serum (Fig. 7, green line). Because of the multitude of serum proteins, it was not possible to ascribe the maxima to the particular HBC-protein complexes. Moreover, the polycations and polyanions also form complexes called polyion complexes (PICs). In our case the respective polycation and polyanion are HBC and HS, which in PBS (i.e. in the absence of proteins) formed complexes smaller than $200 \mathrm{~nm}$ (Fig. 7, black line). The PICs may also interact with proteins to form even larger complexes. ${ }^{53}$ This might explain why the size of PICs in serum was more disperse and extended to greater sizes than PICs in PBS (Fig. 7, black line vs. green line) and why the PICs formed by 


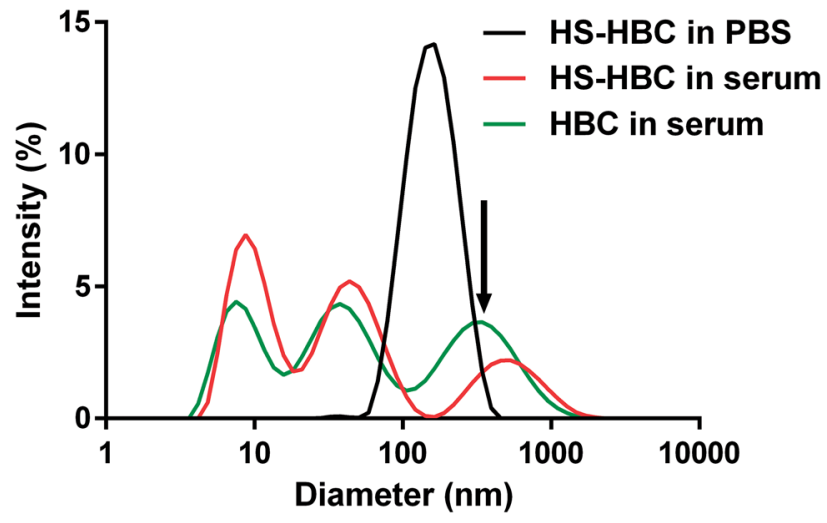

Fig. 7 The size distribution of heparin-binding copolymer-heparan sulfate (HBC-HS) complexes measured by dynamic light scattering (DLS) analysis. The concentrations of $\mathrm{HS}$ and $\mathrm{HBC}$ were $0.4 \mathrm{mg} \mathrm{mL}^{-1}$ and $12 \mathrm{mg} \mathrm{mL}^{-1}$, respectively.

HS and HBC in serum were greater than those in PBS (Fig. 7, red line $v s$. green line).

The measurements of the zeta potential of HS in the systems with increasing concentrations of HBC (Table 1) indicated that the surface charge of the HS-HBC complexes became more and more positive with increasing concentration of HBC. The zeta potential increased monotonically from $-8.6 \mathrm{mV}$ to $+2.3 \mathrm{mV}$ for $\mathrm{HBC} / \mathrm{HS}$ mass ratio in the range of 0.3 to 1.5 . These experiments allowed also to find out that the zeta potential of the complexes at the $\mathrm{HBC} / \mathrm{HS}$ mass ratio corresponding to complete neutralization of anticoagulant activity of HS (about 0.6 ) was equal to $-6.5 \mathrm{mV}$, while their size was about $170 \mathrm{~nm}$. The negative zeta potential of the HS-HBC complexes at this particular mass ratio indicates that these complexes interact mostly with cationic serum proteins.

Most importantly, the measurement of the size of HBC-HS complexes indicated that the diameter of the complexes formed in the blood vessels would not exceed $300 \mathrm{~nm}$ (Fig. 7). This is a safe size concerning in vivo use and will not cause congestion in blood vessels.

In the next step, we applied ITC technique to measure the heat of the interaction between HS and HBC. We tried to determine binding affinity, stoichiometry, and enthalpy of in PBS solution and elucidate the mechanisms underlying molecular interactions. The obtained data show that the enthalpy $\Delta H\left(\mathrm{kcal} \mathrm{g}^{-1}\right)$ of interaction was $(9.8 \pm 0.2) \times 10^{-2}$ (Fig. 8) confirming the formation of the complex. The ITC measurement indicated that the process of HS complexation by HBC continued until the HBC/HS mass ratio reached about 1 , what is indicated by the sudden drop of the evolved heat occurring at this ratio down to values close to zero. Since the measurement gave the net thermal effect of the interaction of

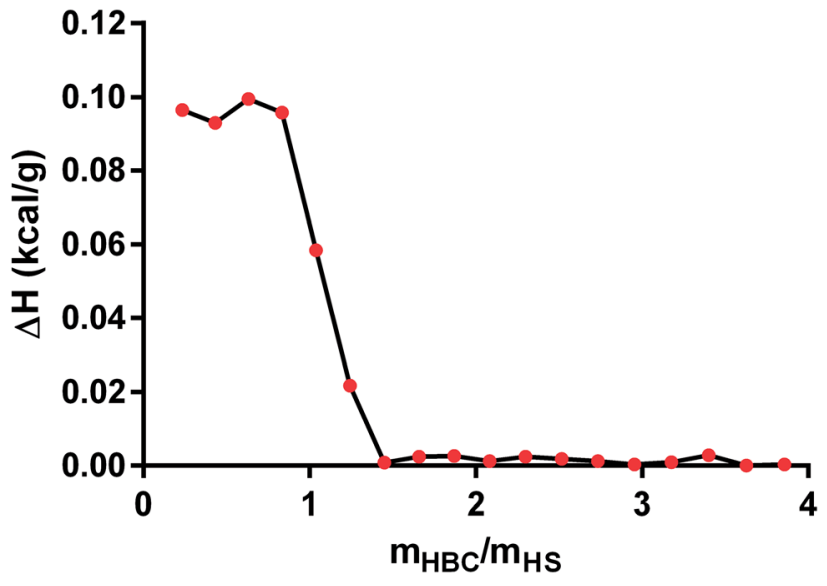

Fig. 8 The estimated heat of binding for the titration of heparan sulfate $(\mathrm{HS})$ with heparin-binding copolymer ( $\mathrm{HBC}$ ) measured by isothermal titration calorimetry (ITC) method.

HS with HBC (the solvent was the same for both polymers so the heat of mixing of both solutions was zero) it could be concluded that further addition of HBC did not result in the formation of complexes. Comparison of these results with those obtained from the measurement of the zeta potential indicated also that at HBC/HS mass ratio of 1 the zeta potential of the complexes reached zero. Thus, the positive net zeta potential of aggregates observed at $\mathrm{HBC} / \mathrm{HS}$ mass ratio above 1 resulted from the excess of positively charged unbound $\mathrm{HBC}$ macromolecules present in the solution together with the HS-HBC complexes whose zeta potential was close to zero.

\section{Discussion}

In the present study, we aimed to check if $\mathrm{HBC}$ can bind and neutralize HS. We showed that HBC binds and forms complexes with HS, and as a result, it reverses all the tested parameters changed by HS. Importantly, the reversal of the anticoagulant activity of HS by HBC was also observed in the living organism.

The binding of HS and HBC was characterized by a colorimetric Azure A method, ITC and DLS techniques in the saline or serum. We found that HBC binds this HS by forming complexes below $200 \mathrm{~nm}$ with less than $1: 1$ stoichiometry. The size of complexes is important for the safety issues: particles higher than $1000 \mathrm{~nm}$ may block the smallest human pulmonary arterioles. The neutralization was confirmed in vitro, and then in vivo by measuring aPTT, PT, anti-fXa activity, anti-fIIa activity, and platelet aggregation. HBC reversed the enhancement of all tested parameters caused by HS.

We used HS with $N$-acetylglucosamine $\left(A_{\mathrm{Nac}}\right)$ content of $25 \%$ (which was almost twice higher than that of heparin), as found

Table 1 The zeta potential and the average size of complexes formed at different heparin-binding copolymer/heparan sulfate (HBC/HS) mass ratios found from dynamic light scattering (DLS) measurements

\begin{tabular}{lcccccc}
\hline HBC/HS mass ratio & 0 (pure HS) & 0.3 & 0.6 & 0.75 & 0.9 & 1.2 \\
\hline Zeta potential [mV] & $-10.3 \pm 0.7$ & $-8.6 \pm 1.4$ & $-6.5 \pm 0.1$ & $-4.7 \pm 0.6$ & $-2.7 \pm 0.8$ & $0.9 \pm 0.1$ \\
Z-average size [nm] & $<10.0$ & 89.0 & 169.7 & 154.0 & 143.8 & 371.3
\end{tabular}


from HS-QC NMR spectra (data not shown) while the content of iduronic acid 2-O-sulphate groups was $45 \%$ which can be compared with $74 \%$ for heparin and $21 \%$ for HS reported by Guerrini $^{54}$ suggesting that the HS we have studied was quite a highly sulfated variant of this GAG.

We chose these coagulation parameters because it is welldocumented that they provide useful information related to HS and HS-mimetic monitoring. ${ }^{\mathbf{1 , 5 5 , 5 6}}$ The anticoagulant activity of HS, although different to UFH, is also mediated via its ability to activate the inhibition of factor $\mathrm{Xa}$ and factor IIa by antithrombin (AT).,55 Others showed that the significant prolongation of aPTT by HS was achieved at concentrations or doses much higher than those of UFH. Similar effects, in favor of UFH, were obtained on the inactivation of factor IIa and factor Xa by AT. ${ }^{55,57}$ On the other hand, a better safety index was observed for HS compared to UFH. ${ }^{\mathbf{5 8 , 5 9}}$ Similarly to others, ${ }^{\mathbf{1 , 5 5 , 5 9}}$ we found the maximum anticoagulant effect of HS in plasma at $0.05 \mathrm{mg} \mathrm{mL}^{-1}$ as measured by aPTT, anti-fXa, and anti-fIIa activity tests. The slight differences in the anticoagulant response to HS between our findings and other studies may result from non-identical procedures and structural heterogeneity of HS obtained from various sources and species.

The binding and the inhibition of HS by protamine was previously demonstrated, ${ }^{\mathbf{4 1}}$ but HS was inactive in the Xa assay, unless AT was exogenously added. The neutralization was even weaker in the experimental conditions more closely resembling real blood circulation (in the presence of bovine albumin), and no experiments with protamine were performed in vivo. In the beginning, we checked the binding of HS by HBC in a colorimetric method using Azure A, a cationic dye. The experiment revealed that $\mathrm{HBC}$ completely bound $\mathrm{HS}$ with an optimal ratio of 0.9 : 1. Next, we used well-documented coagulation parameters (aPTT, PT, anti-factor IIa activity, and anti-factor Xa activity) for HS monitoring. The in vitro results of the coagulation tests indicated that the full neutralization of HS required an HBC to HS ratio of about $0.75: 1$. In our recently published studies, ${ }^{\mathbf{4 3 , 4 4}}$ we observed that the degree of inhibition of anticoagulant by antidote in the in vitro studies correlated positively with this degree in the in vivo studies. Therefore, we used the same ratio $(0.75: 1)$ in an animal model. Rats were intravenously injected with HBC (4.5 mg kg-1) after injection with HS $\left(6 \mathrm{mg} \mathrm{kg}^{-1}\right)$ (ratio $0.75: 1$ ). We showed that HBC completely neutralized prolonged aPTT and PT, and increased activity of anti-fXa and anti-fIIa in the living organism. Our results demonstrate that HBC could bind and inhibit HS in humans with higher efficacy than protamine, which ensures that it could be a good drug candidate. Small molecules antagonizing efficiently heparins, such as surfen or ciraparantag, could also neutralize HS, but no such experiments were performed. HBC could have a therapeutic value in bleeding related to $\mathrm{HS}$, which accompany various disorders such as cancer or MPS. Probably, other functions of HS could also be blocked by HBC, as we showed in the platelet aggregation assay. The literature data describing the effect of HS on the platelet aggregation is not evident. GAGs isolated from the bovine aorta (containing only $1.7 \%$ of HS) and HS alone inhibited thrombin-induced platelet aggregation, ${ }^{\mathbf{6 0 , 6 1}}$ but collagen-induced platelet aggregation was not affected.
Danaparoid, a mixture of sulphated glycosaminoglycorunans derived from hog intestinal mucosa, also did not inhibit the release of serotonin from collagen-activated rabbit platelets. ${ }^{62}$ In our study, HS slightly potentiated collagen-induced platelet aggregation. We conducted an experiment in rats treated with HS, not in the exogenous mixture of platelets and HS. There is also evidence showing that heparin may increase collageninduced platelet aggregation. ${ }^{63,64}$ In our experiment, HBC reversed the platelet activating effect of HS. Inhibition of HS activity by HBC is probably a result of binding and formation of inactive HBC-HS complexes as we showed in the colorimetric method with Azure A and ITC technique. We may expect that the virus infection through complexed HS could be interrupted.

We are aware that, besides efficacy, the new drug candidate should possess a beneficial safety profile, ensuring its successful entry into initial clinical phases of development. The example of protamine shows clearly that cationic macromolecules show cytotoxicity, which limits their usefulness as human medicines. ${ }^{42}$ However, HBC was designed to be better tolerated than protamine. HBC, consisting of nonionic PEG block and cationic block PMAPTAC assembles with anionic HS forming PICs when mixed in aqueous solution. PICs are composed of a PEG shell and segregated cation-anion PIC core. ${ }^{46}$ The characteristics of HBC, the neutralization of UFH, enoxaparin and fondaparinux, and the toxicity data were described by us previously. ${ }^{43}$ We showed that $\mathrm{HBC}$, when infused into rats in the therapeutic doses, did not change: blood pressure, heart rate, tissue perfusion of rat's paw, blood oxygen saturation, respiratory rate, peak exhaled $\mathrm{CO}_{2}$, the serum concentration of aspartate aminotransferase, alanine transaminase, alkaline phosphatase, creatine phosphokinase, creatinine and the number of white blood cells, red blood cells, hemoglobin, hematocrit, mean corpuscular volume, mean corpuscular hemoglobin, mean corpuscular hemoglobin concentration or blood platelets. Similarly, in the present manuscript, administration of HBC did not potentiate, but rather inhibited WBC increase induced by HS. Nevertheless, we are still evaluating the nonclinical safety of HBC, and future experiments in the small and large animal models of specific diseases are needed before entering clinical tests.

\section{Conclusions}

In the current study, we presented a new pharmacological tool with a clear target of action (the neutralization of HS). The principle of HS neutralization indicates promising therapeutic potential of HBC in various diseases involving overproduction of HS.

\section{Conflicts of interest}

There are no conflicts to declare.

\section{Acknowledgements}

This research was funded by National Science Centre, Poland grant number 2016/21/B/ST5/00837. The efficacy studies were 
also supported by Medical University of Bialystok project number N/ST/ZB/18/003/2211.

\section{References}

1 N. W. Shworak, T. Kobayashi, A. D. Agostini and N. C. Smits, Prog. Mol. Biol. Transl. Sci., 2010, 93, 153-178.

2 U. Lindahl and L. Kjellén, J. Intern. Med., 2013, 273, 555-571.

3 A. Oldberg, L. Kjellen and M. Hook, J. Biol. Chem., 1979, 254, 8505-8510.

4 U. Lindahl and J. Li, Int. Rev. Cell Mol. Biol., 2009, 276, 105159.

5 M. M. Fuster, L. Wang, J. Castagnola, L. Sikora, K. Reddi, P. H. A. Lee, K. A. Radek, M. Schuksz, J. R. Bishop, R. L. Gallo, P. Sriramarao and J. D. Esko, J. Cell Biol., 2007, 177, 539-549.

6 D. A. Simon Davis and C. R. Parish, Front. Immunol., 2013, 4, 470.

7 M. Forsberg, K. Holmborn, S. Kundu, A. Dagälv, L. Kjellén and K. Forsberg-Nilsson, J. Biol. Chem., 2012, 287, 1085310862.

8 A. Parra, N. Veraldi, M. Locatelli, M. Fini, L. Martini, G. Torri, L. Sangiorgi and A. Bisio, Glycobiology, 2012, 22, 248-257.

9 P. Tátrai, K. Egedi, Á. Somorácz, T. H. Van Kuppevelt, G. Ten Dam, M. Lyon, J. A. Deakin, A. Kiss, Z. Schaff and I. Kovalszky, J. Histochem. Cytochem., 2010, 58, 429-441.

10 S. R. Pallerla, R. Lawrence, L. Lewejohann, Y. Pan, T. Fischer, U. Schlomann, X. Zhang, J. D. Esko and K. Grobe, J. Biol. Chem., 2008, 283, 16885-16894.

11 G. S. Kumar, A. K. Shetty and P. V. Salimath, J. Ethnopharmacol., 2008, 115, 276-283.

12 M. A. Soares, F. C. Teixeira, M. Fontes, A. L. Arêas, M. G. Leal, M. S. G. Pavão and M. P. Stelling, BioMed Res. Int., 2015, 2015, 253801.

13 E. H. Knelson, J. C. Nee and G. C. Blobe, Trends Biochem. Sci., 2014, 39, 277-288.

14 F. Cecchi, D. Pajalunga, C. A. Fowler, A. Üren, D. Rabe, B. Peruzzi, N. MacDonald, D. Blackman, S. Stahl, R. A. Byrd and D. Bottaro, Cancer Cell, 2012, 22, 250-262.

15 K. Fjeldstad and S. O. Kolset, Curr. Drug Targets, 2005, 6, 665-682.

16 C. Ts'ao, T. S. Galluzzo, S. J. Hart, A. S. Ng, P. G. Sorenson and V. Subbarao, Thromb. Haemostasis, 1985, 54, 768-772.

17 M. Nishio, T. Yamanaka, K. Matsumoto, H. Kimura, K. Sakai, A. Sakai, T. Sone, A. Horiike, F. Koizumi, K. Kasahara, T. Ohira, N. Ikeda, N. Saijo, T. Arao and K. Nishio, J. Thorac. Oncol., 2011, 6, 1889-1894.

18 R. N. Palmer, M. E. Rick, P. D. Rick, J. A. Zeller and H. R. Gralnick, N. Engl. J. Med., 1984, 310, 1696-1699.

19 S. A. Khan, R. W. Mason, R. Giugliani, K. Orii, T. Fukao, Y. Suzuki, S. Yamaguchi, H. Kobayashi, T. Orii and S. Tomatsu, Mol. Genet. Metab., 2018, 125, 44-52.

20 J. Tolar, P. J. Orchard, N. S. Key and B. R. Blazar, J. Thromb. Haemostasis, 2008, 6, 893-895.

21 A. Jinno and P. W. Park, Methods Mol. Biol., 2015, 1229, 567585.

22 P. Hilgard and R. Stockert, Hepatology, 2000, 32, 1069-1077.
23 Y. Chen, T. Maguire, R. E. Hileman, J. R. Fromm, J. D. Esko, R. J. Linhardt and R. M. Marks, Nat. Med., 1997, 3, 866-871. 24 T. H.-C. Tang, S. Alonso, L. F.-P. Ng, T.-L. Thein, V. J.-X. Pang, Y.-S. Leo, D. C.-B. Lye and T.-W. Yeo, Sci. Rep., 2017, 7, 46191. 25 V. H. Pomin, F. F. Bezerra and P. A. G. Soares, Curr. Pharm. Des., 2017, 23, 3405-3414.

26 J. Jiang, X. Wu, H. Tang and G. Luo, PLoS One, 2013, 8, e67982.

27 M. Yoon, A. Zago, D. Shukla and P. G. Spear, J. Virol., 2003, 77, 9221-9231.

28 Y.-J. Chien, W.-J. Chen, W.-L. Hsu and S.-S. Chiou, Virology, 2008, 379, 143-151.

29 W. B. Klimstra, K. D. Ryman and R. E. Johnston, J. Virol., 1998, 72, 7357-7366.

30 X. Bai, H. Bao, P. Li, W. Wei, M. Zhang, P. Sun, Y. Cao, Z. Lu, Y. Fu, B. Xie, Y. Chen, D. Li, J. Luo and Z. Liu, Virol. J., 2014, 11, 132.

31 P. Merilahti, E. Karelehto and P. Susi, PLoS One, 2016, 11, e0147168.

32 M. Nasimuzzaman and D. A. Persons, Mol. Ther., 2012, 20, 1158-1166.

33 M. Tamhankar, D. M. Gerhardt, R. S. Bennett, N. Murphy, P. B. Jahrling and J. L. Patterson, Virol. J., 2018, 15, 135.

34 L. Martin, S. Schmitz, R. De Santis, S. Doemming, H. Haase, J. Hoeger, L. Heinbockel, K. Brandenburg, G. Marx and T. Schuerholz, PLoS One, 2015, 10, e0127584.

35 K. F. Hofmann-Kiefer, G. I. Kemming, D. Chappell, M. Flondor, H. Kisch-Wedel, A. Hauser, S. Pallivathukal, P. Conzen and M. Rehm, Eur. J. Med.Res., 2009, 14, 526-531.

36 M. Schuksz, M. M. Fuster, J. R. Brown, B. E. Crawford, D. P. Ditto, R. Lawrence, C. A. Glass, L. Wang, Y. Tor and J. D. Esko, Proc. Natl. Acad. Sci. U. S. A., 2008, 105, 1307513080.

37 M. L. Huang, A. L. Michalak, C. J. Fisher, M. Christy, R. A. A. Smith and K. Godula, Stem Cells, 2018, 36, 45-54.

38 S. M. A. Naini, C. Yanicostas, R. Hassan-Abdi, S. Blondeel, M. Bennis, R. J. Weiss, Y. Tor, J. D. Esko and N. SoussiYanicostas, Transl. Neurodegener., 2018, 7, 6.

39 V. Ferro, Expert Opin. Ther. Targets, 2013, 17, 965-975.

40 R. Kisilevsky, W. A. Szarek, J. Ancsin, R. Vohra, Z. Li and S. Marone, J. Mol. Neurosci., 2004, 24, 167-172.

41 A. R. Hubbard and C. A. Jennings, Thromb. Haemostasis, 1985, 53, 86-89.

42 E. Sokolowska, B. Kalaska, J. Miklosz and A. Mogielnicki, Expert Opin. Drug Metab. Toxicol., 2016, 12, 897-909.

43 B. Kalaska, K. Kaminski, J. Miklosz, S.-I. Yusa, E. Sokolowska, A. Blazejczyk, J. Wietrzyk, I. Kasacka, K. Szczubialka, D. Pawlak, M. Nowakowska and A. Mogielnicki, Transl. Res., 2016, 177, 98-112.

44 B. Kalaska, K. Kamiński, J. Miklosz, K. Nakai, S. I. Yusa, D. Pawlak, M. Nowakowska, A. Mogielnicki and K. Szczubiałka, Biomacromolecules, 2018, 19, 3104-3118.

45 Y. Mitsukami, M. S. Donovan, A. B. Lowe and C. L. McCormick, Macromolecules, 2001, 34, 2248-2256.

46 S.-I. Yusa, Y. Yokoyama and Y. Morishima, Macromolecules, 2009, 42, 376-383. 
47 B. Kalaska, K. Kaminski, E. Sokolowska, D. Czaplicki, M. Kujdowicz, K. Stalinska, J. Bereta, K. Szczubialka, D. Pawlak, M. Nowakowska and A. Mogielnicki, PLoS One, 2015, 10, e0119486.

48 B. Kalaska, K. Kaminski, E. Sokolowska, D. Czaplicki, M. Kujdowicz, K. Stalinska, K. Szczubialka, J. Bereta, D. Pawlak, M. Nowakowska and A. Mogielnicki, in NSTI: Advanced Materials - TechConnect Briefs 2015, 2015, vol. 3.

49 K. Kamiński, M. Płonka, J. Ciejka, K. Szczubiałka, M. Nowakowska, B. Lorkowska, R. Korbut and R. Lach, J. Med. Chem., 2011, 54, 6586-6596.

50 K. Kamiński, B. Kałaska, P. Koczurkiewicz, M. Michalik, K. Szczubiałka, A. Mogielnicki, W. Buczko and M. Nowakowska, MedChemComm, 2014, 5, 489-495.

51 B. Kalaska, E. Sokolowska, K. Kaminski, K. Szczubialka, K. Kramkowski, A. Mogielnicki, M. Nowakowska and W. Buczko, Eur. J. Pharmacol., 2012, 686, 81-89.

52 X. Xu, S. Angioletti-Uberti, Y. Lu, J. Dzubiella and M. Ballauff, Langmuir, 2018, DOI: 10.1021/acs.langmuir.8b01802.

53 S. Young, M. Wong, Y. Tabata and A. G. Mikos, J. Controlled Release, 2005, 109, 256-274.

54 M. Guerrini, A. Naggi, S. Guglieri, R. Santarsiero and G. Torri, Anal. Biochem., 2005, 337, 35-47.
55 A. N. Teien, U. Abildgaard and M. Höök, Thromb. Res., 1976, 8, 859-867.

56 H. Zhou, S. Roy, E. Cochran, R. Zouaoui, C. L. Chu, J. Duffner, G. Zhao, S. Smith, Z. Galcheva-Gargova, J. Karlgren, N. Dussault, R. Y. Q. Kwan, E. Moy, M. Barnes, A. Long, C. Honan, Y. W. Qi, Z. Shriver, T. Ganguly, B. Schultes, G. Venkataraman and T. K. Kishimoto, PLoS One, 2011, 6, e21106.

57 F. A. Ofosu, M. R. Buchanan, N. Anvari, L. M. Smith and M. A. Blajchman, Ann. N. Y. Acad. Sci., 1989, 556, 123-131.

58 P. A. Ockelford, C. J. Carter and J. Hirsh, Pathology, 1985, 17, 78-81.

59 D. Hoppensteadt, J. M. Walenga and J. Fareed, Thromb. Res., 1990, 60, 191-200.

60 Y. Nakashima, T. Matsushima, T. Sadayasu, K. Takahara, J. Segawa and A. Kuroiwa, Artery, 1992, 19, 256-270.

61 G. Luzzatto and R. Paolini, Angiology, 1989, 40, 170-174.

62 D. G. Meuleman, P. M. J. Hobbelen, G. van Dedem and H. C. T. Moelker, Thromb. Res., 1982, 27, 353-363.

63 S. F. Mohammad, W. H. Anderson, J. B. Smith, H. Y. Chuang and R. G. Mason, Am. J. Pathol., 1981, 104, 132-141.

64 J. Hirsh, Am. J. Surg., 1991, 161, 512-518. 